\title{
Avaliação nutricional de refeições e análise de estrutura física em restaurante popular de Sorocaba-SP
}

\author{
Nutritional evaluation of meals and analysis of physical structure of popular \\ restaurant in Sorocaba-SP
}

\author{
Evaluación nutricional de comidas y análisis de la estructura física en popular \\ restaurante de Sorocaba-SP
}

\author{
Rodrigo Lorenzi POLUHA \\ Camila Corrêa MOTTA ${ }^{2}$ \\ Raquel Rosalva GATTI ${ }^{3}$ \\ ${ }^{1}$ Cirurgião Dentista, Mestrando em Odontologia Integrada junto ao Departamento de Odontologia, Universidade Estadual de Maringá-UEM, \\ Maringá-PR, Brasil \\ ${ }^{2}$ Nutricionista graduada pela Universidade Estadual do Centro-Oeste - UNICENTRO (Campus Cedeteg), Guarapuava-PR, Brasil \\ ${ }^{3}$ Professora do Departamento de Nutrição, Universidade Estadual do Centro-Oeste-UNICENTRO (Campus Cedeteg), Guarapuava-PR, Brasil
}

\begin{abstract}
Resumo
O presente trabalho objetiva avaliar o valor nutricional de macro e micronutrientes, servidos durante almoço em um restaurante popular de Sorocaba-SP e analisar a estrutura física do estabelecimento. A coleta de dados foi realizada através de pesagem dos alimentos durante o almoço, seguindo como referência os valores da Dietary Reference Intakes e para a análise da estrutura utilizou-se o check-list da Agencia Nacional de Vigilância Sanitária. Para obtenção dos resultados utilizou-se o software DietWin ${ }^{\circledR}$ e o software SPSS versão 18.0. Obtiveram-se valores superiores a recomendação para: consumo energético $(1.354,80 \pm 158,79 \mathrm{kcal})$; carboidratos $(217,82 \pm 21,23 \mathrm{~g})$; fibra alimentar $(25,17 \pm 11,14 \mathrm{~g})$; proteínas $(54,89 \pm 13,84 \mathrm{~g})$; vitamina C $(124,66 \pm 51,86 \mathrm{mg})$; ferro $(9,50 \pm 4,44 \mathrm{mg})$ e sódio $(1.870,76 \pm 757,75 \mathrm{mg})$. Houve valores inadequados e inferiores para: lipídeos $(29,32 \pm 5,18 \mathrm{~g})$; vitamina A $(134,85 \pm 69,39 \mathrm{mcg})$ e cálcio $(213,06 \pm 118,12 \mathrm{mg})$. Com diferenças estatisticamente significativas $(\mathrm{p}<0,05)$ para fibras, vitamina A, vitamina $\mathrm{C}$, ferro e cálcio. Quanto à estrutura física, 96,8\% dos itens encontravam-se em conformidade. Observou-se inadequação nos nutrientes. São fundamentais a adequação e o balanceamento da comida, principalmente em um restaurante popular devido ao grande número de refeições servidas e pessoas atendidas diariamente.
\end{abstract}

Descritores: Avaliação Nutricional; Macronutrientes; Micronutrientes.

\begin{abstract}
This study aims to evaluate the nutritional value of macro and micronutrients, served during lunch at a popular restaurant of Sorocaba-SP and analyze the physical structure of the establishment. Data collection was performed by weighing the food during lunch, following reference values of the Dietary Reference Intakes and the analysis of the structure used the checklist of the National Health Surveillance Agency. To obtain the results we used the DietWin ${ }^{\circledR}$ software and SPSS software version 18.0. Were obtained values exceeding the recommendation for: energy consumption (1354.80 \pm 158.79 kcal); carbohydrates $(217.82 \pm 21.23 \mathrm{~g})$; dietary fiber $(25.17 \pm 11.14 \mathrm{~g})$; proteins $(54.89 \pm 13,84 \mathrm{~g})$; vitamin C $(124.66 \pm 51.86 \mathrm{mg})$; iron $(9.50 \pm 4.44$ $\mathrm{mg}$ ) and sodium $(1870.76 \pm 757.75 \mathrm{mg})$. There were inadequate and lower values for: lipid (29.32 $\pm 5.18 \mathrm{~g})$; vitamin A (134.85 $\pm 69.39 \mathrm{mcg})$ and calcium $(213.06 \pm 118.12 \mathrm{mg})$. With significant differences ( $\mathrm{p}<0.05)$ fiber, vitamin A, vitamin $\mathrm{C}$, iron and calcium. As for the physical structure, $96.8 \%$ of the items were in compliance. There were inadequate nutrients. They are fundamental the adaptation and balance of food, particularly in a popular restaurant due to the number of meals served and people served daily.
\end{abstract}

Descriptors: Nutrition Assessment; Macronutrients; Micronutrients.

\begin{abstract}
Resumen
Este estudio tiene como objetivo evaluar el valor nutricional de macro y micronutrientes, servidos durante el almuerzo en un restaurante popular en Sorocaba-SP y analizar la estructura física del establecimiento. La recolección de datos se realizó pesando la comida durante el almuerzo, siguiendo como referencia los valores de Dietary Reference Intakes y para el análisis de la estructura fue usada la lista de verificación de la Agencia Nacional de Vigilancia Sanitaria. El análisis de los resultados se realizó con el software de DietWin ${ }^{\circledR}$ y el software SPSS versión 18.0. Se obtuvieron valores superiores a lo recomendado para: consumo energético $(1354,80 \pm 158,79 \mathrm{kcal})$; carbohidratos $(217,82 \pm 21,23 \mathrm{~g})$; fibra dietética $(25,17 \pm 11,14 \mathrm{~g})$; proteínas $(54,89 \pm 13,84 \mathrm{~g})$; vitamina C $(124,66 \pm 51,86 \mathrm{mg})$; hierro $(9,50 \pm 4,44 \mathrm{mg})$ y sodio $(1870,76 \pm 757,75 \mathrm{mg})$. Se encontraron valores inadecuados e inferiores para: lípidos $(29,32 \pm 5,18 \mathrm{~g})$; vitamina A $(134,85 \pm 69,39 \mathrm{mcg})$ y calcio (213,06 $\pm 118,12 \mathrm{mg}$ ). Con diferencias estadísticas significativas $(\mathrm{p}<0,05)$ para fibras, vitamina A, vitamina C, hierro y calcio. En cuanto a la estructura física, el $96,8 \%$ de los ítems cumplían con las condiciones. Los nutrientes estaban inadecuados. Es fundamental la adaptación y balanceo de los alimentos, especialmente en un restaurante popular debido al gran número de comidas servidas y de personas atendidas diariamente.
\end{abstract}

Descriptores: Evaluación Nutricional; Macronutrientes; Micronutrientes. 


\section{INTRODUÇÃO}

Alimentação adequada é fundamental, para garantir o desenvolvimento, a promoção de saúde e o bem-estar dos indivíduos ${ }^{1,2}$. A qualidade de uma refeição é diretamente relacionada aos valores nutricionais que ela contempla ${ }^{3}$. Dentro deste contexto, entende-se que os mais variados estabelecimentos de fornecimento de refeições necessitam estar devidamente preparados, buscando refeições equilibradas e de qualidade ${ }^{4}$.

Restaurantes Populares são Unidades de Alimentação e Nutrição (UAN) que têm como princípios fundamentais a produção e a distribuição de refeições saudáveis, com alto valor nutricional, feitas com produtos regionais, a preços acessíveis, servidas em locais apropriados e confortáveis, de forma a garantir a dignidade do ato de se alimentar. Sua gestão é responsabilidade do poder público local e a produção mínima estimada para um serviço desta natureza é de mil refeições diárias no horário do almoço, por no mínimo, cinco dias da semana ${ }^{5}$.

Esses restaurantes são destinados a oferecer à população que se alimenta fora de casa refeições variadas, mantendo o equilíbrio entre os nutrientes (proteínas, carboidratos, sais minerais, vitaminas, fibras e água) em uma mesma refeição, possibilitando ao máximo o aproveitamento pelo organismo, reduzindo os grupos de risco à saúde ${ }^{5,6}$.

Para a garantia dessa adequação nutricional é necessário estabelecer se a média do que é consumido pelos clientes acata as necessidades nutricionais dos indivíduos, segundo as recomendações da Dietary Reference Intakes (DRI's), para cada faixa etária e sexo ${ }^{7}$, bem como se a estrutura física dos espaços da produção das refeições contempla o recomendado pelo check-list da Agência Nacional de Vigilância Sanitária ${ }^{8}$.

O objetivo desse trabalho consistiu em avaliar o valor nutricional de macro e micronutrientes servidos durante almoço em restaurante popular de Sorocaba-SP, bem como analisar a estrutura física do mesmo.

\section{MATERIAL E MÉTODO}

A coleta de dados para a avaliação do consumo alimentar foi realizada durante cinco dias nos almoços oferecidos aos usuários, em um restaurante popular, localizado município de Sorocaba-SP. Inicialmente realizou-se a pesagem das preparações servidas pelos funcionários com os respectivos utensílios utilizados pela UAN, por meio do uso de balança digital (Toledo Prix ${ }^{\circledR} 3$ Light $15 \mathrm{Kg}$ ). Após a pesagem do prato o mesmo era entregue de forma aleatória para um usuário do restaurante.

Os cardápios foram avaliados a partir das quantidades da porção de todos os alimentos e preparações fornecidos pelo restaurante. $\mathrm{Na}$ avaliação da composição nutricional da refeição, foi analisado o valor energético, o percentual de energia proveniente dos macronutrientes (carboidratos, proteínas e lipídeos), assim como os principais micronutrientes (ferro, cálcio, vitamina $\mathrm{C}$, vitamina A e sódio).

A avaliação dos cálculos nutricionais foi realizada a partir da média das refeições em um período de cinco dias. Posteriormente, foi realizado o cálculo de adequação do almoço, para compará-la às recomendações diárias de acordo com o Guia Alimentar para a População Brasileira, determinando o Valor Calórico Total/:ia ${ }^{2}$.

Os dados obtidos foram calculados com base no software DietWin ${ }^{\circledR}$ Profissional 2008, porém, quando o alimento não estava listado no programa, foram utilizados os dados da Tabela de Composição Química dos Alimentos ${ }^{9-11}$.

Foram avaliadas questões relativas a aspectos higiênicos sanitários apenas para a estrutura física do restaurante, utilizando-se um check-list de 62 itens, elaborado pela ANVISA ${ }^{8}$. Esta análise ocorreu por meio de observações no próprio local durante a coleta de dados, as opções de respostas foram: "Adequado" (A), quando o restaurante se apresentou em conformidade ao item observado e "Não Adequado" (NA), quando não estava de acordo.

A análise estatística realizou-se por meio de percentagens, médias e desvio-padrão por meio do software Microsoft Excel®. Utilizou-se também o pacote estatístico Statistical Package for the Social Sciences (SPSS) versão 18.0 para tabulação e análise estatística dos dados de forma descritiva, bem como para o teste estatístico com um nível de significância de 5\% $(\mathrm{p}<0,05)$. O teste "T" de Student pareado foi utilizado para verificar se existe diferença significativa entre as variáveis quantitativas do estudo.

\section{RESULTADOS}

O restaurante popular servia no máximo 1200 refeições ao dia e somente no horário do almoço. Os cardápios, durante os dias de coleta, foram compostos por um tipo de salada, um prato principal, três a quatro tipos de guarnições (arroz e feijão e pão de leite fixos), uma sobremesa (fruta ou doce) e um tipo de suco artificial (Tabela 1).

Tabela 1. Preparações servidas na UAN dispostas

\begin{tabular}{|c|c|c|c|c|c|}
\hline Dia & Salada & $\begin{array}{c}\text { Prato } \\
\text { Principal }\end{array}$ & Guarnições* & Sobremesa & Suco \\
\hline $2^{a}$ feira & Repolho & $\begin{array}{l}\text { Bolinho de } \\
\text { carne ao } \\
\text { molho de } \\
\text { tomate }\end{array}$ & $\begin{array}{c}\text { Farofa de } \\
\text { cenoura, arroz e } \\
\text { feijão }\end{array}$ & $\begin{array}{l}\text { Doce de geléia } \\
\text { industrializado }\end{array}$ & Abacaxi \\
\hline $3^{\text {a feira }}$ & Alface & $\begin{array}{l}\text { Frango } \\
\text { cozido }\end{array}$ & $\begin{array}{l}\text { Cenoura cozida, } \\
\text { arroz e feijão }\end{array}$ & Banana & Tangerina \\
\hline $4^{\mathrm{a}}$ feira & Tomate & Feijoada & $\begin{array}{c}\text { Farofa de couve } \\
\text { e arroz }\end{array}$ & Laranja & Limão \\
\hline $5^{\text {a feira }}$ & Couve & $\begin{array}{l}\text { Carne } \\
\text { moída }\end{array}$ & $\begin{array}{l}\text { Polenta, arroz e } \\
\text { feijão }\end{array}$ & Laranja & Tangerina \\
\hline $6^{a}$ feira & Acelga & $\begin{array}{c}\text { Coxa e } \\
\text { sobrecoxa } \\
\text { assado }\end{array}$ & $\begin{array}{c}\text { Virado de } \\
\text { abobrinha, arroz } \\
\text { e feijão }\end{array}$ & Pé de moleque & Laranja \\
\hline
\end{tabular}


A quantidade das porções oferecidas para a produção dos pratos servidos no restaurante pode ser observada na Tabela 2.

Foram calculados valores de macro e micronutrientes das refeições oferecidas no restaurante popular pesquisado. (Tabela 3). A Tabela 3 analisa os valores totais de cada nutriente disponíveis nos cardápios de segunda à sexta-feira.

A análise de adequação do consumo de macro e micronutrientes, separando o gênero pode ser observada na Tabela 4.

Tabela 2. Porcionamento das preparações oferecidas pelo Restaurante Ponular nos cinco dias analisados

\begin{tabular}{|c|c|c|c|}
\hline Preparação & $\begin{array}{l}\text { Porção } \\
\text { (g) }\end{array}$ & Preparação & $\begin{array}{l}\text { Porção } \\
\text { (g) }\end{array}$ \\
\hline Arroz branco* & 304 & Salada de acelga & 30 \\
\hline Feijão carioca* & 170 & Salada de alface & 40 \\
\hline Feijoada & 300 & Salada de couve & 40 \\
\hline Frango cozido & 100 & $\begin{array}{c}\text { Salada de repolho } \\
\text { verde }\end{array}$ & 20 \\
\hline Frango assado & 100 & $\begin{array}{l}\text { Salada de tomate } \\
\text { com cebola }\end{array}$ & 120 \\
\hline $\begin{array}{l}\text { Bolinho de carne ao } \\
\text { molho de tomate }\end{array}$ & 220 & Pão de leite & 20 \\
\hline Carne moída & 120 & Banana & 100 \\
\hline Virado de abobrinha & 180 & Laranja & 120 \\
\hline Farofa de couve & 80 & $\begin{array}{l}\text { Doce de geleia } \\
\text { industrializado }\end{array}$ & 20 \\
\hline Farofa de cenoura & 60 & Pé de moleque & 25 \\
\hline Polenta & 120 & Sucos diversos & 200 \\
\hline Cenoura ralada cozida & 80 & & \\
\hline
\end{tabular}

Tabela 3. Análise nutricional das refeições oferecidas nos cinco dias da semana

\begin{tabular}{|c|c|c|c|c|c|c|c|c|c|c|}
\hline Dia & $\begin{array}{l}\text { VET } \\
\text { (Kcal) }\end{array}$ & $\begin{array}{l}\text { Proteína } \\
\text { s (g) }\end{array}$ & $\begin{array}{l}\text { Carboidrat } \\
\text { os (g) }\end{array}$ & $\begin{array}{l}\text { Fibras } \\
\text { (g) }\end{array}$ & $\begin{array}{l}\text { Lipídeos } \\
\text { (g) }\end{array}$ & $\begin{array}{l}\text { Vitamina A } \\
\text { (mcg) }\end{array}$ & $\begin{array}{l}\text { Vitamina C } \\
\text { (mg) }\end{array}$ & $\begin{array}{l}\text { Ferro } \\
\text { (mg) }\end{array}$ & $\begin{array}{l}\text { Cálcio } \\
\text { (mg) }\end{array}$ & $\begin{array}{l}\text { Sódio } \\
\text { (mg) }\end{array}$ \\
\hline$S$ & $1.491,82$ & 58,45 & 243,20 & 25,03 & 31,68 & 46,61 & 46,83 & 12,55 & 208,64 & $3.027,63$ \\
\hline$T$ & $1.187,12$ & 45,12 & 192,45 & 21,52 & 26,31 & 137,08 & 101,20 & 4,99 & 134,28 & $1.120,54$ \\
\hline$Q$ & $1.551,24$ & 77,56 & 228,66 & 43,29 & 36,25 & 185,52 & 142,15 & 15,09 & 415,56 & $1.942,57$ \\
\hline$Q$ & $1.300,51$ & 48,77 & 225,40 & 23,13 & 22,64 & 216,86 & 179,52 & 9,59 & 180,47 & $2.002,97$ \\
\hline $\boldsymbol{S}$ & $1.243,32$ & 44,54 & 199,37 & 12,89 & 29,74 & 88,18 & 153,58 & 5,28 & 126,37 & $1.260,07$ \\
\hline Média & $1.354,80$ & 54,89 & 217,82 & $25,17^{*} 1$ & 29,32 & $134,85^{*}$ & $124,66^{*}$ & $9,50^{*}$ & $213,06^{*}$ & $1.870,76$ \\
\hline$\sigma$ & 158,79 & 13,84 & 21,23 & 1,14 & 5,18 & 69,39 & 51,86 & 4,44 & 118,12 & 757,75 \\
\hline
\end{tabular}

VET = valor energético total. $\sigma=$ Desvio Padrão

* Estatisticamente significante $(\mathrm{p}<0,05)$

Tabela 4. Comparação do valor nutricional das refeições analisadas na UAN em relação às recomendações das DRI's para homens e mulheres

\begin{tabular}{|c|c|c|c|c|c|c|}
\hline & \multicolumn{2}{|c|}{ Gênero Feminino } & \multirow[b]{2}{*}{$\begin{array}{l}\text { Valor } \\
\text { de } p^{* *}\end{array}$} & \multicolumn{3}{|c|}{ Gênero Masculino } \\
\hline & $\underset{*}{\text { Recomendação }}$ & $\begin{array}{c}\text { Adequação } \\
\text { (\%) }\end{array}$ & & $\underset{*}{\text { Recomendação }}$ & $\begin{array}{c}\text { Adequação } \\
\text { (\%) }\end{array}$ & $\begin{array}{l}\text { Valor } \\
\text { de } p^{* *}\end{array}$ \\
\hline $\begin{array}{c}\text { Energia } \\
\text { (kcal) }\end{array}$ & 700 & 193,54 & 0,000 & 700 & 193,54 & 0,000 \\
\hline Proteínas (g) & 16,1 & 340,93 & 0,003 & 19,6 & 280,05 & 0,004 \\
\hline $\begin{array}{c}\text { Carboidratos } \\
\text { (g) }\end{array}$ & 45,5 & 478,72 & 0,004 & 45,5 & 478,72 & 0,004 \\
\hline Fibras (g) & 8,75 & 287,66 & 0,005 & 13,3 & 189,25 & 0,021 \\
\hline Lipídeos (g) & 61,3 & 47,83 & o,ooo & 61,3 & 47,83 & 0,000 \\
\hline Vitamina A & 245 & 55,04 & 0,023 & 315 & 42,81 & 0,004 \\
\hline Vitamina $\mathrm{C}$ & 26,25 & 474,89 & 0,013 & 26,25 & 474,89 & 0,013 \\
\hline Ferro & 6,3 & 150,79 & 0,182 & 2,8 & 339,28 & 0,027 \\
\hline Cálcio & 350 & 60,87 & 0,016 & 350 & 60,87 & 0,016 \\
\hline Sódio & 525 & 356,33 & o,002 & 525 & 356,33 & 0,002 \\
\hline
\end{tabular}


Os itens avaliados seguindo o check-list foram agrupados na Tabela 5 .

Tabela 5. Aspectos e itens avaliados no check-list

\begin{tabular}{|c|c|c|c|}
\hline Aspectos e itens avaliados & $\begin{array}{l}\text { No de }^{\circ} \\
\text { itens }\end{array}$ & $\begin{array}{l}\text { No itens } \\
\text { críticos }\end{array}$ & $\begin{array}{c}\text { \% de } \\
\text { Adequação }\end{array}$ \\
\hline $\begin{array}{l}\text { Edificação e instalações - Área } \\
\text { externa, acesso, área interna, } \\
\text { piso, tetos, paredes e divisórias, } \\
\text { portas, janelas e outras } \\
\text { aberturas, instalações sanitárias } \\
\text { e vestiários para os } \\
\text { manipuladores, instalações } \\
\text { sanitárias para visitantes e } \\
\text { outros, lavatórios na área de } \\
\text { produção, iluminação e } \\
\text { instalação elétrica, ventilação e } \\
\text { climatização, controle integrado } \\
\text { de vetores e pragas urbanas, } \\
\text { manejo dos resíduos e layout. }\end{array}$ & 50 & 6 & $90,3 \%$ \\
\hline $\begin{array}{l}\text { Equipamentos, móveis } \\
\text { utensílios }\end{array}$ & 9 & - & $100 \%$ \\
\hline $\begin{array}{l}\text { Manipuladores - Vestuário e } \\
\text { hábitos higiênicos }\end{array}$ & 3 & - & $100 \%$ \\
\hline TOTAL & 62 & 6 & $96,8 \%$ \\
\hline
\end{tabular}

\section{DISCUSSÃO}

De acordo com o Guia Alimentar para a População Brasileira $^{2}$ o percentual para o almoço deve ser de $35 \%$ do total de nutrientes de todas as refeições/dia, sendo destacada a importância do fracionamento da dieta pelas refeições, utilizando volumes pequenos com adaptações individuais levando em consideração as atividades profissionais $^{11}$.

O consumo energético médio encontrado no almoço foi de $1.354,80 \pm 158,79 \mathrm{kcal}$, superior ao recomendado de 700 calorias de acordo com o preconizado para uma dieta de $2000 \mathrm{kcal}^{11}$. A ingestão média de carboidratos esteve acima do ideal, sendo ingeridos pelos usuários do restaurante uma quantia de $217,82 \pm 21,23 \mathrm{~g}$ e o que se recomenda é de $45,5 \mathrm{~g}$, tanto para homens como para mulheres. Esses valores elevados são recorrentes na literatura ${ }^{12}$, sendo preocupante, uma vez que esse excesso poderá desencadear patologias como o diabetes mellitus, dislipidemias e obesidade ${ }^{13,14}$.

Outro achado excedente foi o teor de fibra alimentar, que atingiu uma média de $25,17 \pm 11,14 \mathrm{~g}$, com uma adequação de $287,66 \%$ e $189,25 \%$, para mulheres e homens, respectivamente, estando acima dos valores recomendados, sendo esta divergência estatisticamente significante $(\mathrm{p}<0,05)$. O efeito benéfico das fibras é relacionado ao controle do diabetes mellitus, prevenção e tratamento da doença do cólon e até mesmo redução do risco de câncer ${ }^{15,16}$.

Percebe-se que para proteínas, foi encontrado um

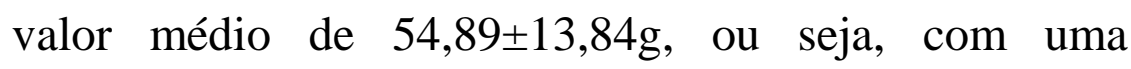
adequação de $340,93 \%$ e $280,05 \%$ para mulheres e homens, respectivamente, quando comparada com a recomendação da DRI. Desta forma, o consumo de fontes proteicas no almoço deveria ser reduzido, visto que uma dieta rica em proteína pode ocasionar problemas renais e até mesmo aumentar o risco de osteoporose $^{17}$.

Entre os macronutrientes, apenas a ingestão média de lipídeos foi inferior à recomendação, sendo ingerido $29,32 \pm 5,18 \mathrm{~g}$ e o recomendado é $61,3 \mathrm{~g}$, apresentando uma inadequação de 56,17\%. Este achado contraria outros trabalhos com alta proporção de lipídios ${ }^{18,19}$. Essa divergência é possivelmente explicada pelas distintas porções utilizadas em cada estudo.

Em relação aos micronutrientes analisados, a vitamina A atingiu um valor médio de $134,85 \pm 69,39 \mathrm{mcg}$, com uma adequação de 55,04\% para homens e $42,81 \%$ para mulheres. A ingestão média de vitamina $C$ foi de $124,66 \pm 51,86 \mathrm{mg}$, estando acima da preconizado para o almoço que seria de $26,25 \mathrm{mg}$, para ambos os gêneros, assim, obteve uma adequação de $474,89 \%$ quando comparados com a DRI. Savio et al. ${ }^{20}$, em pesquisa realizada por participantes do programa de alimentação do trabalhador, revela valores de vitamina $\mathrm{C}$ de 34,3 e $27,7 \mathrm{mg}$, para mulheres e homens, respectivamente. A vitamina $\mathrm{C}$ possui pouca toxidade ao organismo, e quando administrada em altas doses, após atingir concentração máxima nos tecidos, sofre eliminação do excesso pelos rins ${ }^{21}$. Em ambas vitaminas a divergência dos valores em relação aos preconizados foi estatisticamente significante $(\mathrm{p}<0,05)$.

A quantidade média de ferro encontrada na pesquisa foi de $9,50 \pm 4,44 \mathrm{mg}$, com uma adequação de $150,79 \%$ para mulheres e $339,28 \%$ para homens. O ferro tem um papel importante no organismo, sua deficiência é considerada um dos maiores problemas de saúde pública, devido à prevalência da anemia ${ }^{22}$. Encontrou-se um valor médio de cálcio de 213,06 $\pm 118,12 \mathrm{mg}$, ou seja, estando assim abaixo do ideal, que seria de $350 \mathrm{mg}$ para o almoço, com uma inadequação de $39,13 \%$ quando comparados ao preconizado. Estimular um adequado consumo de cálcio parece ser uma importante estratégia de prevenção em relação à osteoporose ${ }^{23}$. Para os dois itens, foi estatisticamente significante $(\mathrm{p}<0,05)$ a discrepâncias dos índices ideais.

O sódio encontrou-se acima da recomendação $1.870,76 \pm 757,75 \mathrm{mg}$, quando comparados às recomendações da DRI, com uma adequação de $356,33 \%$ para ambos os gêneros. Essa quantidade de sódio encontrada é atribuída somente aos alimentos que continham esse nutriente. Não foi computado o sódio referente ao sal do preparo da refeição e da adição em que cada usuário poderia utilizar no momento da refeição. A restrição de sal na dieta é uma medida apoiada para a população de um modo geral e pode também reduzir em longo prazo, o risco de problemas cardiovasculares $^{24,25}$.

Quanto à avaliação dos itens do check-list, observou-se que em relação aos aspectos de edificação e instalações obteve $90,3 \%$ ( $n=44)$ de adequação conforme 
preconizado pela Resolução $\mathrm{RDC} / 275$ enquanto que apenas 9,7\% (n=6) não estavam adequados. Os aspectos avaliados sobre os equipamentos, móveis, utensílios e manipuladores, se encontravam dentro das conformidades. O check-list é uma ferramenta que permite fazer uma avaliação preliminar das condições higiênico-sanitárias de um estabelecimento de produção de alimentos, permitindo levantar pontos críticos e a partir dos dados coletados, traçarem ações corretivas para adequação de instalações, procedimentos e processos produtivos, buscando eliminar ou reduzir riscos físicos, químicos e biológicos, que possam comprometer os alimentos e a saúde do consumidor ${ }^{8}$.

Os dados apresentados por este estudo mostram que, apesar de o almoço oferecido pelos restaurantes populares atender às necessidades energéticas e de macronutrientes, nota-se um consumo médio de calorias, carboidratos e proteínas, fibras, ferro e sódio dos usuários acima da recomendação. Além disso, constatouse carência no consumo lipídeos e de alguns micronutrientes, como é o caso da vitamina A e o cálcio. Uma das formas para se atingir um alto padrão de qualidade dos alimentos é a implantação das Boas Práticas de Fabricação (BPF). Estas são compostas por um conjunto de princípios e regras para o correto manuseio de alimentos, que abrange desde a recepção das matérias-primas até o produto final, o seu principal objetivo é garantir a integridade do alimento e a saúde do consumidor $^{26,27,28}$

\section{CONCLUSÃO}

De acordo com os resultados encontrados na análise dos cardápios dos cincos dias avaliados, observou-se inadequação de todos os nutrientes, de acordo com os valores padrões recomendados pela DRI. Quanto à estrutura física, a maioria dos itens avaliados encontrava-se em conformidade. Conclui-se que as UAN, devido a quantidade elevada de porções das preparações oferecidas e do número de pessoas que atendem, devem fazer adequação nutricional a fim de prover e contribuir para hábitos alimentares saudáveis.

\section{REFERÊNCIAS}

1. Brasil. Ministério da Saúde. Política Nacional de Alimentação e Nutrição. Brasília. 2013. Disponível em: http://bvsms.saude.gov.br/ bvs/publicacoes/ politica_nacional_alimentacao_nutricao.pdf.

2. Brasil. Ministério da Saúde. Secretaria de Atenção à Saúde. Guia alimentar para a população brasileira: promovendo a alimentação saudável. Brasília. 2008. Disponível em: http://bvsms.saude.gov.br/ bvs/ publicacoes/guia_alimentar_populacao_brasileira_20 08.pdf

3. Branquinho A, Sávio KEO, Akutsu RCCA, Silva E F. Sociodemographic and health profi le of clients of community restaurants of brazilian social programs. Rev Chil Nutri. 2015; 42(1):14-22.

4. Godoy, KCr; Sávio, KEO; Akutsu, RCCA; Gubert, MB; Botelho, RBA. Perfil e situação de insegurança alimentar dos usuários dos Restaurantes Populares no Brasil. Cad Saúde Pública. 2014; 30(6):1239-49.

5. Ministério do Desenvolvimento Social e Combate à Fome: Manual Programa Restaurante Popular. Brasília, 2004. Disponível em: http://bvsms.saude.gov.br/bvs/publicacoes/projeto_lo gico_restaurante_popular.pdf

6. Araújo TG, Botelho RBA, Akutsu RCCA, Araújo Wilma MC. Conformity of food service units with legislation. J Culinary Sci \& Technol. 2015; 14: 7589.

7. Food and Nutrition Board. Dietary Reference Intakes (DRI): Macronutrients. SOURCES: Dietary Reference Intakes for Energy, Carbohydrate. Fiber, Fat, Fatty Acids, Cholesterol, Protein, and Amino Acids. Institute of Medicine. 2005. Disponível em: https://fnic.nal.usda.gov/sites/fnic.nal.usda.gov/files/ uploads/energy_full_report.pdf.

8. Brasil. Ministério da Saúde. Agência Nacional de Vigilância Sanitária. RDC- 275. Regulamento técnico sobre procedimentos operacionais padronizados aplicados aos estabelecimentos produtores/industrializadores de alimentos e a lista de verificação das boas práticas de fabricação em estabelecimentos produtores/industrializadores de alimentos. 2002. Disponível em: http:// portal.anvisa.gov.br/wps/wcm/connect/dcf7a900474 $576 \mathrm{fa} 84 \mathrm{cfd} 43 \mathrm{fbc} 4 \mathrm{c} 6735 / \mathrm{RDC}+\mathrm{N} \% \mathrm{C} 2 \% \mathrm{BA}+275,+\mathrm{D}$ $\mathrm{E}+21+\mathrm{DE}+\mathrm{OUTUBRO}+\mathrm{DE}+2002 . \mathrm{pdf} ? \mathrm{MOD}=\mathrm{AJPE}$ RES

9. Carvalho FMA, Amorim SAA, Silva MMS, Gomes ST. Investimento Social e Perfil dos Usuários do Primeiro Restaurante Popular de Belo Horizonte MG. Reuna. 2007; 12(2):21-37.

10. Franco G. Tabela de composição química dos alimentos. 9a ed. Rio de Janeiro: Atheneu; 2002.

11. Guimarães AF, Galisa MS. Cálculos Nutricionais: Conceitos e aplicações práticas. São Paulo: M. Books; 2008.

12. Oliveira CS, Reis CS, Miranda TS, Akutsu RCCA, Savio KEO, Botelho RBA. Análise da densidade energética de preparações servidas em uma Unidade de Nutrição. Nutrire. 2010; 35(3):77-86.

13. Botelho RBA, Rego, K. Desenvolvimento e aceitabilidade de sobremesas para diabéticos e sua inserção no cardápio de um hospital público de Brasília/DF. Nutrição Brasil. 2013;12: 206-11.

14. Souza FA, Silva RCO, Fernandes CE. Avaliação nutricional de cardápios em unidades de alimentação e nutrição: adequação ao programa de alimentação do trabalhador. Rev Eletr Ciên. 2009; 1(2):43-50. 
15. Mattos LL, Martins IS. Consumo de fibras alimentares em população adulta. Rev Saúde Pública. 2000; 34(1):50-5.

16. Geraldo APG, Bandoni DH, Jaime PC. Aspectos dietéticos das refeições oferecidas por empresas participantes do Programa de Alimentação do Trabalhador na Cidade de São Paulo, Brasil. Rev Panam Salud Publica. 2008; 23(1):19-25.

17. Oliveira CS, Alves FS. Educação nutricional em unidade de alimentação e nutrição, direcionada para consumo de pratos protéicos: um estudo de caso. Alim. Nutr. 2008; 19(4):435-40.

18. Oliveira OMV, Zandonadi RP, Gandolfi L, Almeida RC, Almeida LM, Pratesi R. Evaluation of the Presence of Gluten in Beans Served at Self-Service Restaurants: A Problem for Celiac Disease Carriers. J Culinary Sci \& Technol. 2014; 12(1):22-33.

19. Höfelmann DA, Riekes BH, Azevedo LC. Hábito alimentar e estado nutricional: fatores de risco cardiovascular entre colaboradores internos de uma Unidade de Alimentação e Nutrição. Nutr Pauta. 2005; 70:42-5.

20. Savio KEO, Costa THM, Miazakib E, Schmitz BAS. Avaliação do almoço servido a participantes do programa de alimentação do trabalhador. Rev Saúde Pública. 2005; 39(2):148-155.

21. Aranha FQ, Barros ZF, Moura LSA, Gonçalves MCR, Barros JC, Metri JC et al. O papel da vitamina C sobre as alterações orgânicas no idoso. Rev Nutr. 2000; 13(2):89-97.

22. Ybarra LM, Costa, NMB, Ferreira, CLLF. Interação cálcio e ferro: uma revisão. Nutrire. 2001; 22:85-107.

23. Sichieri R, Coitinho DC, Monteiro JB, Coutinho WF. Recomendações de Alimentação e Nutrição Saudável para a População Brasileira. Arq Bras Endocrinol Metab 2000; 44(3):227-32.

24. Zandonadi R P, Botelho RBA, Ginani VC, Akutsu RCCA, Savio KEO, Araújo WMC. Sodium and health: New proposal of distribution for major meals. Health. 2014; 6(3):195-201.

25. Salas, CKTS et al. Teores de sódio e lipídios em refeições almoço consumidas por trabalhadores de uma empresa do município de Suzano, SP. Rev Nutr. 2009; 22(3):331-9.

26. Akutsu RC, Botelho RBA, Camargo EB, Savio KEO, Araújo WC. Adequação das boas práticas de fabricação em serviços de alimentação. Rev Nutr. 2005; 18(3):419-27.

27. SENAC/DN. Guia passo a passo: Implantação de Boas Práticas e Sistema APPCC. Qualidade e Segurança alimentar. Projeto APPCC Mesa. Convênio CNC/CNI/SEBRAE/ANVISA. Rio de Janeiro; 2001.

28. SENAC/DN. Guia de Verificação. Boas Práticas e Sistema APPCC. Qualidade e Segurança alimentar. Projeto APPCC Mesa. Convênio
CNC/CNI/SEBRAE/ ANVISA. Rio de Janeiro; 2002; p.39-54.

\section{CONFLITO DE INTERESSES}

Os autores declaram não haver conflitos de interesse.

\section{AUTOR PARA CORRESPONDÊNCIA}

Rodrigo Lorenzi Poluha

rodrigopoluha@gmail.com
Submetido em 11/08/2016 Aceito em 12/09/2016 